\title{
Utility of passive photography to objectively audit built environment features of active transport journeys: an observational study
}

\author{
Melody Oliver ${ }^{*}$, Aiden R Doherty ${ }^{2,5}$, Paul Kelly ${ }^{2}$, Hannah M Badland ${ }^{3}$, Suzanne Mavoa ${ }^{3}$, Janine Shepherd ${ }^{1}$,
} Jacqueline Kerr ${ }^{4}$, Simon Marshall ${ }^{4}$, Alexander Hamilton ${ }^{2}$ and Charlie Foster ${ }^{2}$

\begin{abstract}
Background: Active transport can contribute to physical activity accumulation and improved health in adults. The built environment is an established associate of active transport behaviours; however, assessment of environmental features encountered during journeys remains challenging. The purpose of this study was to examine the utility of wearable cameras to objectively audit and quantify environmental features along work-related walking and cycling routes.

Methods: A convenience sample of employed adults was recruited in New Zealand, in June 2011. Participants wore a SenseCam for all journeys over three weekdays and completed travel diaries and demographic questionnaires. SenseCam images for work-related active transport journeys were coded for presence of environmental features hypothesised to be related to active transport. Differences in presence of features by transport mode and in participant-reported and SenseCam-derived journey duration were determined using two-sample tests of proportion and an independent samples t-test, respectively.

Results: Fifteen adults participated in the study, yielding 1749 SenseCam images from 30 work-related active transport journeys for coding. Significant differences in presence of features were found between walking and cycling journeys. Almost a quarter of images were uncodeable due to being too dark to determine features. There was a non-significant tendency for respondents to under-report their journey duration.

Conclusion: This study provides proof of concept for the use of the SenseCam to capture built environment data in real time that may be related to active transportation. Further work is required to test and refine coding methodologies across a range of settings, travel behaviours, and demographic groups.
\end{abstract}

Keywords: Walking, Cycling, SenseCam, Measure, Physical activity

\section{Introduction}

Active transport (e.g., walking or cycling for travel) has been shown to reduce the risk of obesity, hypertension, type 2 diabetes, cardiovascular disease, and overall mortality [1-6]. Active transport provides the opportunity to regularly engage in physical activity that is integrated into daily routines [1,7], and can potentially overcome time constraints, a commonly cited barrier to physical activity engagement in adults [8]. A body of research shows

\footnotetext{
* Correspondence: melody.oliver@aut.ac.nz

'Human Potential Centre, National Institute for Public Health and Mental Health Research, Auckland University of Technology, Auckland, New Zealand Full list of author information is available at the end of the article
}

associations between active transport and built environment characteristics (e.g., presence of sidewalks/footpaths, mixed use, safety features, etc.) [9-11], with time and travel distances facilitating or inhibiting the behaviour $[1,12,13]$.

Much of the research to date has utilised self-reported measures of active transport journeys (e.g., travel diaries) rather than objective measures of travel and/or the environment [9]. Inaccuracies of self-reported travel behaviours have been well documented [14,15]. Recent technological advancements, such as accelerometers, global positioning systems (GPS) units, and geographic information systems (GIS), show promise for objectively assessing the geographical location and characteristics of physical activity

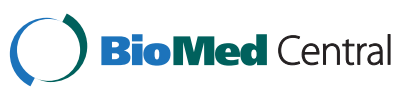


participation [16]. Moreover, photography through wearable camera technologies (e.g., SenseCam) is increasingly being seen as valuable for eliciting environmental information from the participants' perspective [17]. This method reduces reliance on participant recall and reporting of environmental features, and facilitates the detailed description of environmental factors, including aesthetics, context, and quality. The capture of such information from a first person point of view at the time of exposure (e.g., the 'greenness' of walking environments, traffic volumes, temporary obstructions to cycling) cannot be replicated by current alternative objective methods [18].

Manually coding and classifying these environmental features is prohibitively time consuming and costly to implement, even in small-scale research (e.g., <10 participants). Accordingly, new methodologies are being developed to automate treatment of the photographic data [19]. In order for processes to be automated, machine-learning algorithms need to be developed that can correctly classify images that have been manually "ground-truthed" (i.e., using direct observation or expert opinion). The algorithm can then be used to code images in the absence of human verification. The current study details findings from this first stage of research, by examining a proof of concept for the utility of SenseCamderived photographic data to enable the objective quantification of built environment features along work-related walking and cycling routes (active transport journeys). Data can be used to develop a classification system for environmental features captured using passive photography that may be important for classifying environmental features of active travel and encouraging or discouraging active transportation behaviours.

\section{Methods}

\section{Protocol and measures}

This was an observational study conducted in Auckland, New Zealand, in June 2011 (winter). Convenience sampling was utilised to invite fifteen adult employees from two universities to participate in the study. Consenting participants were provided with a SenseCam device and requested to wear the unit on a lanyard around their neck for all journeys over three weekdays. They were also asked to record information about their journeys over this time using a 3 day travel diary based on the United Kingdom Department for Transport National Travel Survey [20]. Demographic information was collected via pen-and-paper questionnaire. Ethical approval was provided by the Host Institution ethics committee (AUTEC 11/114, May 25th 2011).

The SenseCam is a small $(6 \times 7 \times 1.5 \mathrm{~cm})$, lightweight (approx 175g), wearable camera, fitted with a wide-angle (fish-eye) lens [21]. When worn on a lanyard around the neck, first-person point-of-view images from the wearer are captured. The SenseCam contains an internal clock, tri-axial accelerometer, magnetometer, light-intensity and light-colour sensors, a passive infrared (body heat) detector, and a temperature sensor. The camera automatically takes images when triggered by changes in the sensor information collected [21]. On average, this results in image capture every 10 seconds during travel behaviour [22]. When not triggered by sensor data, images are automatically captured every 50 seconds. Images can also be manually captured by the wearer, but not viewed as there is no viewfinder.

Potential environmental features that may be captured from SenseCam images were defined a-priori from a suite of commonly used environmental audits in active transport research as follows: Neighborhood Walking Environment Scale (NEWS) [23]; Systematic Pedestrian and Cycling Environmental Scale (SPACES) [12]; and the Walking and Bicycling Suitability Assessment Forms (WABSA-W and WABSA-B, respectively) [24]. Additional features that were identified as potential influences of active transport that are not captured in these audits were also identified and included (e.g., weather and temporary obstructions to walking or cycling). In total, 30 common active transportrelated environmental features were identified that may potentially be identified from SenseCam images (Table 1).

Numerous privacy and ethical issues exist when using Passive Automated Digital Image-capture (PADI) devices such as the SenseCam. Considerations include passive versus purposive data collection, intrusiveness, informed consent, privacy issues (e.g., data collection in sensitive situations), mitigation of loss of confidentiality, and data collection from unconsenting third parties. The protocols and procedures of the current study adhered to the ethical framework proposed by Kelly et al. for use of PADI devices in research [25].

\section{Data treatment}

Descriptive information for participant demographics was calculated. Body Mass Index (BMI) was determined from self-reported height and weight as follows: weight in kilograms/height in meters ${ }^{2}$. World Health Organization thresholds for overweight and obesity were then applied ( $\geq 25 \mathrm{~kg} / \mathrm{m}^{2}$ and $\geq 30 \mathrm{~kg} / \mathrm{m}^{2}$, respectively) [26]; SenseCam data were downloaded into the Oxford CLARITY SenseCam Browser software (freely available at http://sensecambrowser. codeplex.com). Participants were shown how to browse images and were provided the opportunity to review and delete any images in private if they wished to do so. This SenseCam Browser software automatically groups images into a series of distinct events utilising the accelerometer data [27]. Active transport journeys to or from workplace were identified using a combination of participant-reported journeys derived from the travel diaries, SenseCam-derived events, and manual scanning of SenseCam images. Focusing 
Table 1 Description of environmental features present in walking and cycling journeys

\begin{tabular}{|c|c|c|c|c|c|c|c|}
\hline \multirow[t]{2}{*}{ Feature } & \multirow[t]{2}{*}{ Description } & \multicolumn{2}{|c|}{$\begin{array}{l}\text { Cycling } \\
\left(n=599^{+}\right)\end{array}$} & \multicolumn{2}{|c|}{$\begin{array}{l}\text { Walking } \\
\left(\mathrm{n}=1150^{\dagger}\right)\end{array}$} & \multicolumn{2}{|c|}{$\begin{array}{c}\text { Total } \\
\left(\mathrm{n}=1749^{\dagger}\right)\end{array}$} \\
\hline & & $\mathrm{n}$ & (\%) & $\mathrm{n}$ & (\%) & $\mathrm{n}$ & (\%) \\
\hline Bus stop & Bus stop visible in photo & 44 & 7.3 & 69 & 6.0 & 113 & 6.5 \\
\hline Cars driving & Cars in motion or in traffic lanes on road & 388 & 64.8 & 674 & $58.6^{*}$ & 1062 & 60.7 \\
\hline Cars in carpark & $\begin{array}{l}\text { Cars parked in car park wholly or more than } \\
2 / 3 \text { partially visible }\end{array}$ & 68 & 11.4 & 110 & 9.6 & 178 & 10.2 \\
\hline Cars parked & Cars parked on side of the road & 190 & 31.7 & 151 & $13.1^{* *}$ & 341 & 19.5 \\
\hline Commercial & Commercial or institutional buildings visible & 281 & 46.9 & 648 & $56.3^{* *}$ & 929 & 53.1 \\
\hline Congested traffic & More than 6 stationary cars in driving lanes & 4 & 0.7 & 10 & 0.9 & 14 & 0.8 \\
\hline Cycle lanes & Designated cycle lane on road or footpath & 16 & 2.7 & 247 & $21.5^{* *}$ & 263 & 15.0 \\
\hline Cyclists & Any person/people riding cycles other than the participant & 6 & 1.0 & 8 & 0.7 & 14 & 0.8 \\
\hline Dark & $\begin{array}{l}\text { Image indicates journey conducted in darkness } \\
\text { (e.g., dusk or dawn, streetlights on) but features still } \\
\text { visible and image codeable }\end{array}$ & 120 & 20.0 & 209 & 18.2 & 329 & 18.8 \\
\hline Dogs & Dogs or a lead in participant hand visible & 0 & 0.0 & 4 & 0.3 & 4 & 0.2 \\
\hline Footpath & Footpath visible (not walkway/pathway) & 338 & 56.4 & 761 & $66.2^{* *}$ & 1099 & 62.8 \\
\hline Footpath good condition & No cracks or potholes visible & 327 & 54.6 & 759 & $66.0^{* *}$ & 1086 & 62.1 \\
\hline Graffiti & Graffiti visible & 0 & 0.0 & 2 & 0.2 & 2 & 0.1 \\
\hline Grass verge & Any area of grass either beside road or footpath & 270 & 45.1 & 504 & 43.8 & 774 & 44.3 \\
\hline Grass verge maintained & No obvious weeds or overgrown grass & 262 & 43.7 & 454 & 39.5 & 716 & 40.9 \\
\hline Litter & Litter present (e.g., paper, food wrappings, etc.) & 1 & 0.2 & 1 & 0.1 & 2 & 0.1 \\
\hline Other lights & Lights from houses, buildings or cars in photos & 247 & 41.2 & 348 & $30.3^{* *}$ & 595 & 34.0 \\
\hline Pedestrian crossing & Zebra crossings and traffic light pedestrian crossings visible & 82 & 13.7 & 240 & $20.9^{* *}$ & 322 & 18.4 \\
\hline Pedestrians & Any person/people in the photo other than the participant & 63 & 10.5 & 272 & $23.7^{* *}$ & 335 & 19.2 \\
\hline $\begin{array}{l}\text { Permanent obstructions } \\
\text { to cycling }\end{array}$ & Tree, signage, or other permanent structure in cycleway & 2 & 0.3 & 0 & 0.0 & 2 & 0.1 \\
\hline $\begin{array}{l}\text { Permanent obstructions } \\
\text { to walking }\end{array}$ & $\begin{array}{l}\text { Tree, signage, or other permanent structure on } \\
\text { footpath/walkway }\end{array}$ & 2 & 0.3 & 0 & 0.0 & 2 & 0.1 \\
\hline Rain & Rain visible & 63 & 10.5 & 54 & $4.7^{* *}$ & 117 & 6.7 \\
\hline Residential & Private homes visible & 155 & 25.9 & 229 & $19.9^{* *}$ & 384 & 22.0 \\
\hline Retail buildings & Buildings with retail/shop-fronts visible & 141 & 23.5 & 165 & $14.3^{* *}$ & 306 & 17.5 \\
\hline Road good condition & No cracks or potholes visible & 462 & 77.1 & 820 & $71.3^{* *}$ & 1282 & 73.3 \\
\hline Street lighting & Street lights visible (not including traffic lights) & 209 & 34.9 & 531 & $46.2^{* *}$ & 740 & 42.3 \\
\hline $\begin{array}{l}\text { Temporary obstructions } \\
\text { to cycling }\end{array}$ & Rubbish bins, parked cars, roadworks, etc. in cycleways & 9 & 1.5 & 11 & 1.0 & 20 & 1.1 \\
\hline $\begin{array}{l}\text { Temporary obstructions } \\
\text { to walking }\end{array}$ & $\begin{array}{l}\text { Rubbish bins, parked cars, roadworks, etc. on } \\
\text { footpath/walkway }\end{array}$ & 14 & 2.3 & 41 & 3.6 & 55 & 3.1 \\
\hline Trees & Any trees visible in photo including from a distance & 441 & 73.6 & 842 & 73.2 & 1283 & 73.4 \\
\hline Walkway & $\begin{array}{l}\text { Journey occurring in walkway/pathway } \\
\text { (not road or footpath) }\end{array}$ & 45 & 7.5 & 200 & $17.4^{* *}$ & 245 & 14.0 \\
\hline
\end{tabular}

Notes: Data were collected in Auckland, New Zealand, in June 2011.

$\mathrm{n}=$ number of images.

${ }^{*} \mathrm{p}<0.05 ;{ }^{* *} \mathrm{p}<0.01$ significant difference in features present between walking and cycling journeys; ${ }^{\dagger}$ If photo was too dark to code individual features then it was coded as uncodeable and not included here; \%, percentage of walking or cycling images where feature was present; $n$, number of images.

on journeys between home and workplace only was a pragmatic decision to ensure manageability of data treatment and acknowledging the significant contribution work-related travel makes to overall travel behaviours. Travel diaries were considered the criterion for occurrence and mode of trips undertaken. Although GPS data can be used to identify walking and cycling journeys, trip purpose is not captured, therefore the travel diary was deemed an appropriate measure of work-related journey occurrence for the purposes of the current study. SenseCam images were considered the 
criterion for journey start and end times, which were determined using the protocol of Kelly et al. [22] and active transport events (journeys) for walking or cycling set accordingly. Where trip-chaining occurred (identified from the SenseCam images as stopping at one or more locations between home and work (e.g., shops or cafe), separate events were set for the active transport components of these journeys. Descriptive data (e.g., image filename, date, time) was then extracted as a .csv file for walking or cycling journeys between home and work only using the SQL database manager (http://sensecambrowser.codeplex.com/ documentation), and saved in Microsoft Excel 2007 (Microsoft Corp, Redmond, WA).

Individual trip-chain events were collated into their respective active transport journeys. Participant-reported journeys of less than 10 minutes duration were excluded from analyses. Evidence suggests that a minimum bout of 10 minutes of self-reported physical activity is required for health benefits $[28,29]$. Delimiting data treatment to journeys of at least 10 minutes duration was thus a practical approach to maintaining data treatment manageability and ensuring that data retained were relevant to the field of physical activity and health research. Active transport components of mixed mode journeys (e.g., parking the car and walking) were only retained where they met the 10 minute data inclusion threshold. Individual images were then scanned for each feature listed in Table 1 and given a binary code of 1 or 0 to indicate whether the feature was present or not, respectively. Inter-rater reliability was calculated for $10 \%$ of randomly selected images using Cohen's kappa $(\kappa)$ statistic [30]. Descriptive data were calculated for frequency of features present by travel mode. To examine content validity, differences in absence or presence of features by transport mode were determined using two-sample tests of proportion. Differences in participant-reported and SenseCam-derived journey duration were calculated using an independent samples t-test. Analyses were undertaken using STATA IC 10.1 (StataCorp, TX). Statistical significance was set at $\alpha=0.05$.

\section{Results}

All fifteen participants provided informed consent to participate in the study. Table 2 shows the descriptive information for participant characteristics. One participant did not complete his travel diary, five reported no active transport journeys, and one reported one non workrelated active journey. A total of 44 work-related active transport journeys were captured from the remaining eight participants over the three days. Three trips were removed from further analysis; two because they were reported to be less than 10 minutes duration, and one because there was no corresponding SenseCam data for the reported journey in the travel diary (the SenseCam
Table 2 Participant characteristics (n (\%) unless stated otherwise)

\begin{tabular}{lll}
\hline Variable & $\mathbf{n}$ & $\mathbf{( \% )}$ \\
\hline Age $($ mean $(\mathrm{SD}))$ & 38.6 & $(10.7)$ \\
Sex & & $(20.0)$ \\
\hline Male & 3 & $(80.0)$ \\
Female & 12 & \\
BMI & & $(60.0)$ \\
\hline Normal/Underweight $\left(<25 \mathrm{~kg} / \mathrm{m}^{2}\right)$ & 9 & $(40.0)$ \\
Overweight $\left(\geq 25 \mathrm{~kg} / \mathrm{m}^{2}\right)$ & 6 & $(0.0)$ \\
Obese $\left(\geq 30 \mathrm{~kg} / \mathrm{m}^{2}\right)$ & 0 & $(6.7)$ \\
Occupation & & $(6.7)$ \\
\hline Professor & 1 & $(46.7)$ \\
Senior Lecturer & 1 & $(26.7)$ \\
Researcher & 7 & $(6.7)$ \\
Research administrator/assistant & 4 & $(6.7)$ \\
Teaching assistant & 1 & \\
Technician & 1 & $(20.0)$ \\
Site & & 3 \\
\hline Central city & 12 & \\
Suburban & &
\end{tabular}

Notes: Data were collected in Auckland, New Zealand, in June 2011. BMl, Body Mass Index $\left(\mathrm{kg} / \mathrm{m}^{2}\right)$; n, number of participants; SD, Standard Deviation.

was delivered after the journey was reported). There were 10 instances of trip-chaining, involving 21 separate events (one trip-chain comprised three events). After collating trip-chains into individual journeys, the remaining sample included 30 work-related active transport journeys (yielding 2292 images in total). Almost a quarter $(23.7 \%, \mathrm{n}=$ 543) of images were uncodeable due to being too dark to determine features, a proportion that was relatively similar for walking and cycling trips $(23.1 \%$ and $24.8 \%$ uncodeable, respectively). In total, 1749 photos were coded for environmental features. An example of images captured and corresponding coding is provided in Figure 1.

Significant differences in presence of features were found between walking and cycling journeys, in directions that would be expected (e.g., greater proportion of footpaths and pedestrians found for walking journeys compared with cycling journeys; Table 1). Average active transport journey duration was 21.7 minutes and there was a non-significant tendency for respondents to under-report their journey duration, which was greater in cyclists than walkers (Table 3). Inter-rater reliability for presence or quality of features was considered acceptable (range $\mathrm{K}=0.56-0.95$ across all features [31]).

\section{Discussion}

This study provides support for the utility of the SenseCam to capture contextual features within the built environment 


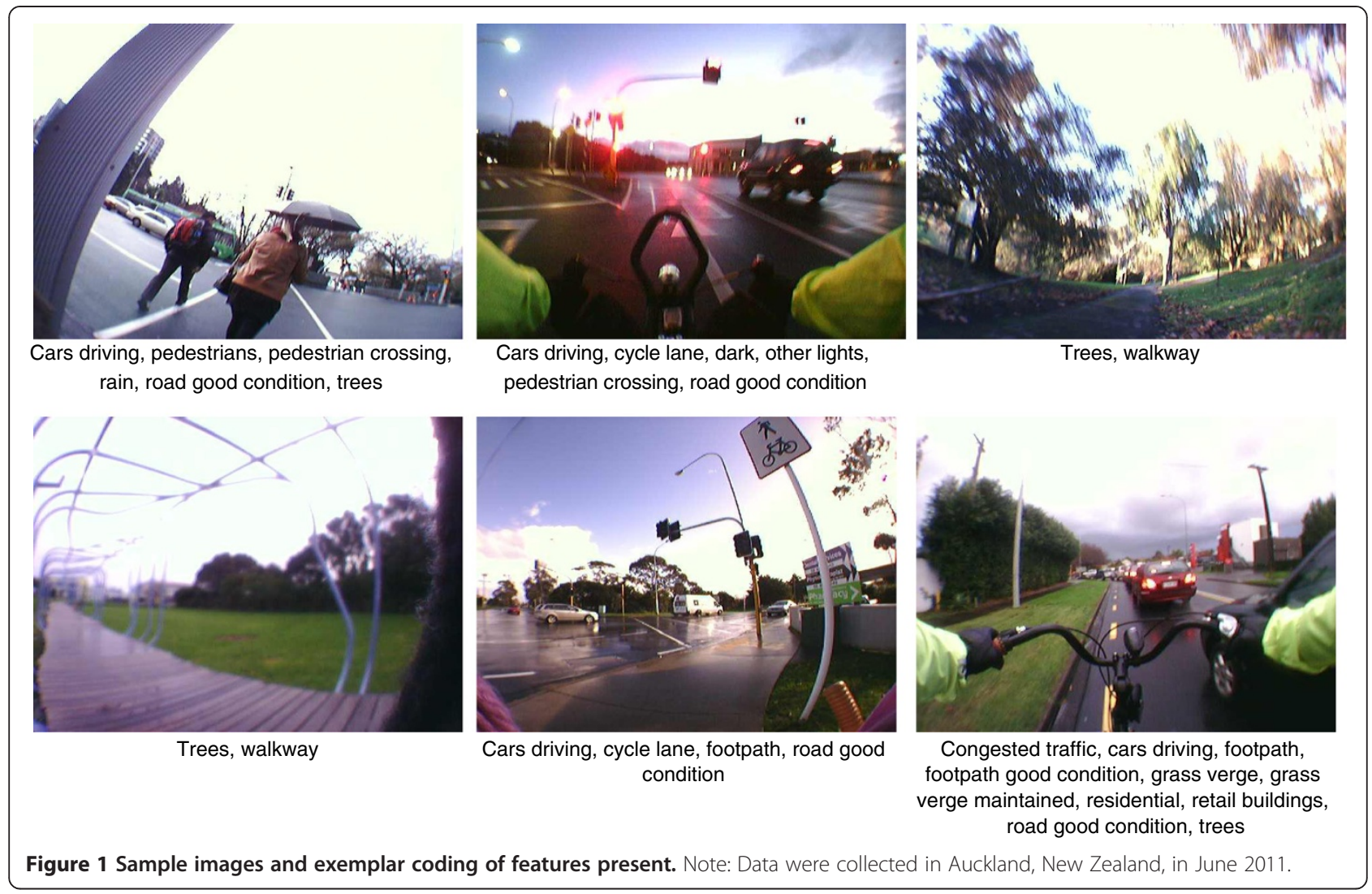

that individuals may encounter during walking or cycling journeys. Although the current dataset was derived from a limited number of participants and geographic area, all hypothesised features of importance identified from the audit tools were identified from the images captured. The tendency to under-report journey duration is in contrast with previous SenseCam research [22,32] and Global Positioning System (GPS) studies [33], possibly due the small convenience sample and focus on work related walking and cycling trips only. We found significant differences in the presence of specific features between walking and cycling modes, suggesting preliminary support for the content validity of this approach. For example, a significantly greater proportion of footpaths, pedestrians, and pedestrian crossings were found for walking trips, while a higher prevalence of car presence was found for cycling journeys. With the exception of cycle lanes, all significant differences between features identified by walking and cycling were in the expected direction. The lack of cycle lanes in the study areas may explain this finding somewhat, whereby many cycling journeys were completed on roads without cycle lanes. Improving on existing audits that do not reflect temporal exposure, use of the SenseCam data enabled the capture of factors that individuals actually encountered during active transport journeys, such as traffic density; weather conditions; presence of pedestrians, cyclists, and dogs; and temporary obstructions to walking or cycling.

Almost a quarter of data were lost due to images being too dark to enable coding of features. In part, this is likely due to the study being conducted during winter, with

Table 3 Journey duration characteristics

\begin{tabular}{|c|c|c|c|c|c|c|}
\hline \multirow[t]{2}{*}{ Trip duration (minutes) } & \multicolumn{2}{|c|}{ Cycling $(n=8)$} & \multicolumn{2}{|c|}{ Walking $(n=21)$} & \multicolumn{2}{|c|}{ Total $(n=29)$} \\
\hline & Mean & $(\min , \max )$ & Mean & $(\min , \max )$ & Mean & $(\min , \max )$ \\
\hline Reported duration & 20.1 & $(15.0,53.0)$ & 22.0 & $(10.0,45.0)$ & 21.5 & $(10.0,53.0)$ \\
\hline SenseCam duration & 21.3 & $(9.9,56.6)$ & 22.3 & $(9.6,60.0)$ & 21.7 & $(9.6,56.6)$ \\
\hline Difference (Reported - SenseCam) & -1.1 & $(-3.6,5.2)$ & -0.2 & $(-11.1,8.6)$ & -0.42 & $(-11.1,8.6)$ \\
\hline
\end{tabular}

Notes: Data were collected in Auckland, New Zealand, in June 2011. 
reduced daylight hours and work-related travel occurring in reduced light. In some instances participants may have intentionally or unintentionally worn the SenseCam with the lens facing inwards or worn an item of clothing over the SenseCam, which would also result in uncodeable images. Researchers may benefit from asking participants whether there were instances where the SenseCam lens was intentionally obscured to account for this. A high proportion of work-related journeys were omitted due to the use of motorised transport modes. Again, this may be due to the study being conducted during winter with weather conditions discouraging active transport modes. It is also likely that some participants resided in environments that were unsupportive for actively transporting to their workplaces, however we cannot establish this from the current investigation.

While employment of photography in health research is not a new concept, the use of wearable cameras to passively capture a series of images over specified times has only been possible in recent years. As we have observed with other emerging technologies in health behaviour research such as pedometry, accelerometry, and GPS, there is a significant amount of research that is first required to develop appropriate and agreed-upon data treatment methods. As noted earlier, this study was conducted with a small sample and was limited to two areas of Auckland, New Zealand, only. Our aim was not to provide a comprehensive framework for coding environmental features, but to provide proof of concept and baseline data for future active transport work across international sites. Research is now needed to determine criterion and predictive validity of SenseCam image coding of environmental features over a range of settings and situations (e.g., heavy traffic) and utilising the wide range of validated environmental audits available. SenseCam images can provide repeated measures of environmental variables encountered during journeys, which may differ by individual, and by journey duration, purpose, and mode. As such it is possible that some journeys or individuals may bias findings (e.g., due to having a greater number of repeated measures of one factor). Future research should thus consider accounting for clustering of environmental features both within journeys and individuals when investigating differences between environmental features encountered. Manual coding of the data was time consuming, taking approximately 25 researcher hours to process the 2292 images (equivalent to approximately 6.4 hours of journey time). Consequently, automated concept detection techniques need to be extended to identify environmental features of interest in future research with larger sample sizes [19]. The wide range of kappa values found for interrater reliability across factors may denote issues with researcher interpretation (such as features being in 'good condition') or difficulties in clearly establishing features (due to photos being blurry for example). Future work to establish clear coding instructions and training protocols for researchers is thus required. Further research is also needed to consider more detailed built environment features than those presented here, for example types of pedestrian crossings, which may be especially important for vulnerable populations. Walking and cycling were the only travel modes examined in the current study; future research should consider the implications of differing travel modes and travel behaviours on image quality (e.g., running may result in blurry/uncodeable images), across a wider range of journey purposes and demographic groups.

\section{Conclusions}

This study provides proof of concept for the use of the SenseCam to capture data on built environment features that may be related to active transportation. Having these temporal contextual data to support environmental audits or GIS-derived built environment measures may improve sensitivity of measures and improve our ability to establish exposures and explain individual preferences for transport mode and routes taken. Considerable opportunities exist to harness the in-depth contextual information on built environments that are captured by SenseCam. This study provides the first step towards understanding these opportunities in relation to active transport.

\section{Abbreviations \\ BMI: Body mass index; NEWS: Neighborhood walking environment scale; PADI: Passive automated digital image-capture; SPACES: Systematic pedestrian and cycling environmental scale; WABSA-B: Bicycling suitability assessment form; WABSA-B: Walking suitability assessment form.}

\section{Competing interest}

The authors have no conflicts of interest to declare.

\section{Authors' contributions}

$\mathrm{MO}, \mathrm{ARD}, \mathrm{PK}, \mathrm{HMB}$, and SM conceived and undertook the study. JS carried out the SenseCam data treatment. MO drafted the manuscript and performed the statistical analysis. JK, SM, AH, and CF participated in the design of the study. All authors contributed to drafting the manuscript, and have read and approved the final manuscript.

\section{Acknowledgements}

This research was supported by an Auckland University of Technology Faculty of Health and Environmental Sciences summer studentship. The authors acknowledge the time of the participants and Ms Moea Genet. AD is a Marie Curie postdoctoral research fellow supported by the Irish Health Research Board under grant number MCPD/2010/12. CF is funded by the British Heart Foundation under grant number 021/P\&C/Core/2010/HPRG. PK is supported by Microsoft Research through its PhD Scholarship Programme.

\section{Author details}

${ }^{1}$ Human Potential Centre, National Institute for Public Health and Mental Health Research, Auckland University of Technology, Auckland, New Zealand. ${ }^{2}$ British Heart Foundation Health Promotion Research Group, Department of Public Health, University of Oxford, Oxford, United Kingdom. ${ }^{3}$ McCaughey VicHealth Centre for the Promotion of Mental Health and Community Wellbeing, the University of Melbourne, Melbourne, Australia. ${ }^{4}$ San Diego State University, San Diego, USA. ${ }^{5}$ CLARITY: Centre for Sensor Web Technologies, Dublin City University, Dublin, Ireland. 
Received: 24 January 2013 Accepted: 2 April 2013

Published: 10 April 2013

\section{References}

1. Badland H, Schofield G, Garrett N: Travel behavior and objectively measured urban design variables: associations for adults traveling to work. Health Place 2008, 14:85-95.

2. Hayashi T, Tsumura K, Suematsu C, Okada K, Fuji S, Endo G: Walking to work and the risk for hypertension in men: the Osaka health survey. Ann Intern Med 1999, 130:21-26.

3. Hu G, Pekkarinen H, Hanninen O, Tian H, Guo Z: Relation between commuting, leisure time physical activity and serum lipids in a Chinese urban population. Ann Hum Biol 2001, 28:412-421.

4. Hu G, Pekkarinen H, Hanninen O, Yu Z, Guo Z, Tian H: Commuting, leisuretime physical activity, and cardiovascular risk factors in China. Med Sci Sports Exerc 2002, 34:234-238.

5. Hu FB, Li TY, Colditz GA, Willett WC, Manson JE: Television watching and other sedentary behaviors in relation to risk of obesity and type 2 diabetes mellitus in women. J Am Med Assoc 2003, 289:1785-1791.

6. Hamer $M$, Chida $Y$ : Active commuting and cardiovascular risk: a metaanalytic review. Prev Med 2008, 46:9-13.

7. Bull FC, Gauvin L, Bauman A, Shilton T, Kohl HW, Salmon A: The Toronto charter for physical activity: a global call for action. J Phys Act Health 2010, 7:421-422

8. Sullivan C, Oakden J, Young J, Butcher H, Lawson R: Obstacles to action: A study of New Zealanders' physical activity and nutrition. Wellington, New Zealand: Sport and Recreation New Zealand; 2003.

9. Badland H, Schofield G: Transport, urban design, and physical activity: an evidence-based update. Transportation Res 2005, Part D:177-196.

10. Transportation Research Board and Institute of Medicine of the National Academies: Does the Built Environment Influence Physical Activity? Examining the Evidence. Washington, DC: Transportation Research Board, Institute of Medicine of the National Academies; 2005

11. Saelens BE, Sallis JF, Frank LD: Environmental correlates of walking and cycling: findings from the transportation, urban design, and planning literatures. Ann Behav Med 2003, 25:80-91.

12. Pikora TJ, Giles-Corti B, Kkuiman MW, Bull FC, Jamrozik K, Donovan RJ: Neighborhood environmental factors correlated with walking near home: using SPACES. Med Sci Sports Exerc 2006, 38:708-718.

13. Duncan M, Mummery W: GIS or GPS? A comparison of the two methods for assessing route taken during active transport. Am J Prev Med 2007 33:51-53.

14. Golob TT, Meurs H: Biases in response over time in a seven-day travel diary. Transportation 1986, 13:163-181.

15. Clarke $M$, Dix $M$, Jones P: Error and uncertainty in travel surveys. Transportation 1981, 10:105-126.

16. Oliver M, Badland HM, Mavoa S, Duncan MJ, Duncan JS: Combining GPS, GIS and accelerometry: methodological issues in the assessment of location and intensity of travel behaviors. J Phys Act Health 2010, 7:102-108.

17. Wang C, Burris MA: Photovoice: concept, methodology, and use for participatory needs assessment. Health Educ Behav 1997, 24:369-387.

18. Weber K: Mobile Devices and a New Understanding of Presence. Copenhagen, Denmark: Falko Schmid, Tobias Hesselmann, Susanne Boll, Keith Cheverst, Lars Kulik (Chairs); 2010.

19. Doherty AR, Caprani N, Conaire CÓ, Kalnikaite V, Gurrin C, Smeaton AF, O'Connor NE: Passively recognising human activities through lifelogging. Comput Hum Behav 2011, 27:1948-1958.

20. Department for Transport: National Travel Survey 2009: Statistical release. London: Author: 2010.

21. Hodges S, Williams L, Berry E, Izadi S, Srinivasan J, Butler A, Smyth G, Kapur N. Wood K: SenseCam: A Retrospective Memory Aid. In UbiComp: 8th International Conference on Ubiquitous Computing vol 4602 of LNCS. Berlin, Heidelberg: Springer; 2006:177-193.

22. Kelly P, Doherty A, Berry E, Hodges S, Batterham AM, Foster C: Can we use digital life-log images to investigate active and sedentary travel behaviour? Results from a pilot study. Int J Behav Nutr Phys Act 2011, 8:44.

23. Cerin E, Saelens BE, Sallis JF, Frank LD: Neighborhood environment walkability scale: validity and development of a short form. Med SC Sports Exerc 2006, 38:1682-1691.

24. Emery J, Crump C: The WABSA Project: Assessing and Improving Your Community's Walkability \& Bikeability. North Carolina: Department of Health
Behavior and Health Education, School of Public Health, The University of North Carolina at Chapel Hill; 2003.

25. Kelly P, Marshall S, Badland H, Kerr J, Oliver M, Doherty AR, Foster C: Ethics of using Passive Automated Digital Image-capture (PADI) devices in health behaviour research. Am J Prev Med 2013, 44:314-319.

26. World Health Organization: Obesity: Preventing and Managing the Global Epidemic: Report of a WHO Consultation. Geneva, Switzerland: Author; 2004.

27. Doherty AR, Pauly-Takacs K, Caprani N, Gurrin C, Moulin CJA, O'Connor NE, Smeaton AF: Experiences of aiding autobiographical memory using the SenseCam. Human Comput Interact 2012, 27:151-174.

28. Woolf-May K, Kearney EM, Owen A, Jones DW, Davison RC, Bird SR: The efficacy of accumulated short bouts versus single daily bouts of brisk walking in improving aerobic fitness and blood lipid profiles. Health Educ Res 1999, 14:803-815.

29. Murphy $\mathrm{MH}$, Hardman AE: Training effects of short and long bouts of brisk walking in sedentary women. Med Sci Sports Exerc 1998, 30:152-157.

30. Cohen JA: A coefficient of agreement for nominal scales. Educ Psychol Meas 1960, 20:37-46.

31. Landis JR, Koch GG: The measurement of observer agreement for categorical data. Biometrics 1977, 33:159-174.

32. Kelly P, Doherty AR, Hamilton A, Matthews A, Batterham AM, Nelson M, Foster C, Cowburn G: Evaluating the feasibility of measuring travel to school using a wearable camera. Am J Prev Med 2012, 43:546-550.

33. Department for Transport: National Travel Survey 2011 GPS pilot: Summary analysis. London: Author; 2011.

\section{doi:10.1186/1476-072X-12-20}

Cite this article as: Oliver et al:: Utility of passive photography to objectively audit built environment features of active transport journeys: an observational study. International Journal of Health

Geographics 2013 12:20.

\section{Submit your next manuscript to BioMed Central and take full advantage of:}

- Convenient online submission

- Thorough peer review

- No space constraints or color figure charges

- Immediate publication on acceptance

- Inclusion in PubMed, CAS, Scopus and Google Scholar

- Research which is freely available for redistribution

Submit your manuscript at www.biomedcentral.com/submit
C Biomed Central 